\title{
ASPECTS OF MASS LOSS AND ANGULAR MOMENTUM LOSS IN BINARIES CONTAINING COOL COMPONENTS
}

\author{
PETER P. EGGLETON \\ Institute of Astronomy \\ Madingley $R d$ \\ Cambridge CBS OHA \\ United Kingdom
}

\begin{abstract}
Cool stars show evidence of dynamo activity which is stronger with more rapid rotation. Tidal friction in a moderately close binary can be a cause of relatively rapid rotation, so that cool components in such binaries are presumably liable to stronger stellar winds than single cool stars. As a consequence, the binary can be subject to orbital angular momentum loss. Both the mass loss and the orbital angular momentum loss can be on a timescale comparable to nuclear evolution in a red subgiant, or even faster. RS CVn stars probably give the best possibility of measuring these processes, although some observational data are difficult to reconcile with simple theories.

Barium stars, and symbiotics, may both be affected by these processes. They must be the products of evolution of moderately wide binaries, as must such objects as cataclysmic variables. I attempt to define the ranges of zero-age parameters necessary to produce such varied objects. A simplistic model of the distribution of stars brighter than 6 th magnitude (a 'Theoretical Bright Star Catalogue') suggests that for every three Ba stars with a measurable orbit, there should be one main sequence 'Ba star'.
\end{abstract}

It should nowadays be almost an axiom of stellar evolution that any cool star (roughly G or later, $T_{\text {eff }} \lesssim 6000 K$ ) is subject to surface activity, the more so the more rapidly it rotates. The activity manifests itself as radio and $\mathrm{X}$-ray emission, as well as chromospheric and transition-region emission, flaring, and rotational modulation due to starspots. These phenomena, 'activity' for short, appear to be due directly or indirectly to dynamo action, which appears to increase by and large with rotation rate. The activity has as consequences both Mass Loss (ML) and Angular Momentum Loss (AML). The latter is an inevitable concomitant of the former, even in the absence but especially in the presence of a magnetic field. ML from one or more components of a binary will affect the orbital period directly. AML will not necessarily affect the orbit if the two components are relatively far apart, but if they are close enough that at least the active component is subjected to tidal friction, then the orbit as well as the spin of the component will be modified. In the rest of this paper I shall use AML to mean orbital AML by this combination of magnetic braking and tidal friction. 
Direct determinations of ML rates are difficult, even for those luminous cool stars which show signatures of mass loss directly in their spectra. A recent estimate (Judge and Stencel 1991) can be written as

$$
-\dot{m}=10^{-13.6}\left(r^{2} / m\right)^{1.43}, \quad\left(r^{2} / m \gtrsim 10^{2.5}\right)
$$

with $\dot{m}$ in solar masses per year, and $r, m$ in solar units. These authors found that equn (1) fitted their data marginally better than the formula of Reimers (1975),

$$
-\dot{m}=10^{-12.4} r L / m
$$

Interestingly, equn (1) extrapolates surprisingly well to the sun. Mullan et al.(1989) estimated an ML rate for the cool dwarf component of V471 Tau (not in the Hyades, according to Heintz 1991), using the the white dwarf component near eclipse as a probe of the $\mathrm{K}$ dwarf's wind. They obtained a value of $z 1.3 .10^{-11} M_{\odot} / \mathrm{yr}$, which is $\sim 10^{3}$ times greater than solar, presumably because tidal friction enforces rapid rotation in the 0.5 day orbit.

Recently İbanoglu (1990) has reported surprisingly steady rates of period change of certain RS CVn binaries, in a long-term study. The four systems (RT Lac, semidetached; AR Lac, SZ Psc and V471 Tau, detached) showing clear rates of change are all decreasing their periods, on timescales of $\sim 10^{6} \mathrm{yrs}$ for the three subgiant systems and $5.10^{6}$ yrs for the dwarf system. If these really represent a secular trend due to $\mathrm{AML}$, then we would require something like

(a) ML increased by factors of $10^{3}-10^{4}$ above solar, as in V471 Tau

(b) an AML rate relatively larger than the ML rate by similar factors of $10^{3}-10^{4}$, implying an Alfvén radius of $\sim 30-100$ orbital radii.

Many astrophysicists would blink at such large rates. A more widely acceptable hypothesis is that we are seeing fluctuations about a mean which varies much more slowly; yet it is surprising that all four systems show the same sign. Hall (1990) has argued that solar-type cycles on a timescale of decades could produce period changes with signs alternating on the same timescale. The mechanism is that the active star's radius changes in response to magnetic field changes at the subphotospheric level, and the radius changes couple to the orbit via efficient tidal friction. Support for the cyclic point of view comes from a different observation of İbanoglu (1990); he finds a change in the magnitude of V471 Tau which appears to be partly a secular trend of $\sim 0.1 \mathrm{mag} /$ decade, and partly a roughly periodic variation of $\sim 0.1 \mathrm{mag}$ with a quasi-period of 15 yrs (based on an observational span of $18 \mathrm{yrs}$ ). However it is barely conceivable that the secular trend can be sustained indefinitely, and so it seems more probable that even the 'secular' trend is only part of a longer-term cycle. Regrettably, as so often happens in studies of binary period changes, we may have to wait another century for more definite results. The kind of compromise that I would prefer (see below) would be a secular rate of period change due to AML comparable (relatively) to the ML rate, and so requiring an Alfvén radius no larger than the orbital separation, combined with quasi-cyclical fluctuations of $\sim 1$ part in $10^{-5}$ in $P$ on timescales of decades.

Although direct AML rates in active cool binaries do not yet seem definitive, there is I think much firmer evidence for the concept of ML enhanced by a close 
companion. This comes from a small number of (detached) RS CVn systems in which the cooler, more evolved component appears to be significantly less massive than its companion. Examples are RW UMa (Popper 1980) and RZ Eri and Z Her (Popper 1988). The masses, in the order cooler/larger plus hotter/smaller, are $1.45+1.5 M_{\odot}, 1.62+1.68 M_{\odot}$, and $1.31+1.61 M_{\odot}$. Clearly at least $0.05-0.1 M_{\odot}$ has been lost by the initial primary in the first two cases, and much more in the third. Such a substantial amount of mass lost cannot be typical of isolated stars at the base of the giant branch; otherwise very few giants would be able to reach even halfway up the first giant branch.

However the case of $\mathrm{Z}$ Her is rather curious. The luminosity of the hotter, smaller (F5) component is only $5 L_{\odot}$. Comparing this with RZ Cha (Popper 1980), consisting of two evolved F5 stars of $1.51 M_{\odot}$ and $7 L_{\odot}$ each, we see that the the F5 component in Z Her is more massive yet substantially less luminous. No simple concept of stellar evolution can accommodate this, apart from a rather drastically non-solar composition. Curiously, the earlier values of masses for $\mathrm{Z}$ Her $\left(1.10+1.22 M_{\odot} ;\right.$ Popper 1980) were in close agreement both with theoretical models and with other observed masses for similar spectral type. Perhaps (Popper, private communication, this conference) the fact that $\mathrm{Z}$ Her's eclipses are only partial means that its radii are not well determined. If the F5 component is $\sim 25 \%$ larger, its luminosity could be increased to a value comfortably larger than either component of RZ Cha. I believe that a re-investigation of this significant system would be well worth while.

I dwell on Z Her a little because it is such a system which should give much the best empirical estimate of ML enhancement. Theory is much too vague to give a quantitative estimate. Tout and Eggleton (1988a) used the earlier data on $\mathrm{Z}$ Her to infer an enhancement of $\sim 10^{2}$ relative to single stars. But if we fall back on $\mathrm{RZ}$ Eri (Popper 1988), the ML rate must still be enhanced by a factor $\sim 10^{2}$ relative to an extrapolation of equn (1).

Such enhancement will mean that by the time that the primary (by which I mean the initially more massive star) of a typical RS CVn comes to fill its Roche lobe, its mass could be reduced to perhaps half it initial mass. This means that the Rochelobe overflow (RLOF) can be relatively slow and steady, rather than the drastic, dynamic-timescale overflow to be expected if the masses were more closely equal (Paczyński and Sienkiewicz 1972). The timescale might be as slow as nuclear, but I would expect it to be governed by AML at a rate a few times faster than nuclear. On this basis it is easier to understand certain Algols such as AR Mon (K3III + K0III, $0.8+2.7 M_{\odot} ; 21.2 \mathrm{~d} ;$ Popper 1980), which would otherwise have been expected to have had drastic RLOF, perhaps followed by a common-envelope phase and then coalescence. There are several Algols whose present parameters suggest a late Case $\mathrm{B}$ origin, and these are all easier to understand on the hypothesis of enhanced ML (Tout and Eggleton 1988b). But if the binary-enhanced ML process (which we refer to as the Companion-Reinforced Accretion Process, or CRAP) is to work in this way, it is necessary that AML should not be enhanced by too large a factor; otherwise the Roche lobe will shrink down on to the primary so rapidly that it will not be able to lose sufficient mass. We anticipate that the ML rate will be enhanced, by the time the primary is close to its Roche radius, to a timescale several times faster than than the nuclear timescale of the primary, and the AML timescale will 
by then be a little faster than the nuclear timescale.

In view of the fact that dynamo activity is likely to be a chaotic process, it would not be surprising if there were a real variation in the degree of activity, both in the short term and averaged over the long term, of cool components of binaries which were initially identical. Rodonó (this conference) has emphasised the time-variability of stellar activity. Thus it may be rather difficult to predict which zero-age binaries, expected under conservative assumptions to undergo late Case B or late Case C RLOF, should evolve either (i) through drastic RLOF to coalescence, or to a short-period pre-cataclysmic binary, (ii) through mild RLOF (by virtue of CRAP) to an Algol, possibly of rather long period, or (iii) avoid RLOF altogether through substantial ML (whether by CRAP, in binaries with periods of a few hundred days, or just by single-star wind in wider binaries), to end up as detached long-period white dwarf plus main sequence dwarf binaries, such as $\mathrm{Ba}$ star precursors. Eggleton and Tout (1989) suggested that (i) and (ii) are more likely for initial periods less than $\sim 100 \mathrm{~d}$, the actual outcome depending on a combination of initial mass ratio and period, and that (iii) is more likely for longer periods. A further estimate is that for periods between 100 and say $300 \mathrm{~d}$, CRAP will ensure that the primary's envelope will be lost earlier than if it were single, which may possibly prevent s-process enrichment since the core may not evolve quite far enough for shell flashes to contribute much nucleosynthesis; while for longer periods still the primary may lose mass at much the same rate as it would have done had it been single. This is consistent with the suggestion of Boffin and Jorissen (1988) that the transfer of s-process-rich matter in $\mathrm{Ba}$ binaries took place via partial capture of a stellar wind, rather than by RLOF.

Ba stars have a claim to be considered the most important class of binaries to have emerged in the last few decades. This is partly because their putative progenitors are intrinsically common, and partly because they themselves are quite common, i.e. they appear to have to be the fairly normal outcome of evolution in a binary with period in the range 1 - 10 yrs. Griffin (1985) has shown that, contrary to a fairly common view that binary periods are uniformly distributed in $\log P$, there is a considerable increase in the number of spectroscopic binaries per unit interval of $\log P$, as $P$ increases from a few days to $\sim 30$ yrs. This increase is roughly like $P^{0.4}$. A period of $30 y$ rs is, coincidentally, both the upper limit (with a few exceptions) to orbits which have been observed spectroscopically so far by Griffin, and the upper limit expected theoretically, in a conservative picture, for binary interaction by RLOF to take place. The increasing distribution over $\log P$ means that of a!l binaries capable, in a conservative picture, of RLOF, those that should produce $\mathrm{Ba}$ stars are the majority. Although it is clear that a conservative picture does not apply, because even single AGB stars of initial masses $1-3 M_{\odot}$ must lose most or all of their mass by the time the core mass has grown to $\sim 0.6-0.7 M_{\odot}$, some interaction, via stellar wind rather than RLOF, will still take place in the period range $\sim 3-30$ yrs.

A possible picture, but a very tentative one, of evolution of binaries with periods in the range of roughly $100 \mathrm{~d}$ to $30 \mathrm{yr}$ and primary initial masses in the range 1 $3 M_{\odot}$ is the following:

(a) 100d - 1yr: interaction by stellar wind, followed by RLOF (producing a symbiotic); little $\mathrm{Ba}$ enrichment of either primary or secondary, because the primary 
is stripped to the core before the core is massive enough to generate shell flashes; spiral-in a possibility for some initial masses ratios

(b) $1 \mathrm{yr}$ - 3yr: interaction by stellar wind, in some cases followed by RLOF (symbiotic); Ba enrichment of primary and hence secondary; spiral-in a rarer possibilty, but still likely for more extreme initial mass ratios

(c) 3 yr - 10yr: interaction by stellar wind only; Ba enrichment of both components; binary remains wide, i.e. no spiral-in

(d) ₹ 10yr: slight interaction by stellar wind; little or no Ba enrichment of secondary, because too little $\mathrm{Ba}$ rich material is captured; binary remains wide.

It is evident, however, that by processes (b) and (c) one would expect to produce main sequence $\mathrm{Ba}$-rich stars, which would only become proper $\mathrm{Ba}$ stars after the enriched secondary has evolved further to a red giant - except that in a small number of cases where the mass ratio was closely equal to unity originally, the secondary may already be a giant when the primary is an s-processing red supergiant. The problem of the apparent non-existence of main sequence stars with enhaced s-process abundances has been noted by Luck and Bond (1982) and Dominy and Lambert (1983). The former have noted that there are indeed some possible candidates, the $\mathrm{CH}$ subgiants, some of which are hardly evolved beyond the main sequence, but it is my impression that there is nothing like the required number of these, relative to Ba stars.

Following the methodology of Eggleton, Fitchett and Tout (1990), who used a crude model of stellar evolution to model the observed distribution of stars brighter than $V=6.0$ as found in the Bright Star Catalogue (BSC; Hoffleit 1983), I have attempted to construct theoretical BS catalogues in which both classical Ba stars, and their putative s-process-rich main sequence precursors, can be enumerated. Using the recent catalogue of $\mathrm{Lü} \mathrm{(1991)}$ of $\mathrm{Ba}$ stars, I find that there are $37 \mathrm{Ba}$ stars with $V \leq 6.0$, and with Ba-richness parameter greater than or equal to 0.5 . There are several more with milder enrichment $(0.1-0.4)$, but these appear to be rather uncertain.

The assumptions that go into a theoretical BS catalogue were given by Eggleton et al.(1990), so I will not repeat them here in detail. One is that all stars are binaries, with a period distribution, per unit interval of $\log P$, that increases roughly like $P^{0.4}$ up to about $200 \mathrm{yrs}$, but then decreases with about the same (but negative) slope to even longer periods. Another is that the primary's mass is drawn at random from an IMF like that of Miller and Scalo (1979); but that there is some degree of correlation between primary and secondary initial mass, in order to account for the number of moderately massive stars which have companions of similar mass. If the two masses were completely uncorrelated, i.e. if both were drawn independently from the same (Miller-Scalo) IMF, then very few OBA stars indeed would have OBA companions. The birth-rate of stars has been assumed constant over a Galactic lifetime of $12 \mathrm{Gyr}$, and the vertical scale height of the Galactic distribution has been assumed to increase with age. The radial variation of the Galactic distribution was ignored, on the grounds that stars brighter than $V=6.0$ come typically from well within $1 \mathrm{kpc}$ of the sun, on which scale the radial variation of Galactic density is not large.

A typical theoretical BSC contains about 1900 MS + MS pairs, about 1900 RG + MS pairs, about 215 WD + MS pairs, and about $640 \mathrm{WD}+\mathrm{RG}$ pairs. 
The remainder, out of about 4900 pairs, are mostly pairs containing a neutron star (no allowance having been made for the disruption of pairs by a supernova explosion); but a modest number $(\sim 25)$ contain two red giants, for example. I define a theoretical classical Ba binary to be a pair with (a) the primary a white dwarf, (b) the secondary cooler than $6000 \mathrm{~K}$, and less luminous than $1000 L_{\odot}$, (c) an orbital period between 300 and 5000 days, and (d) a radial velocity variation large enough to be measurable, the criterion being a function of spectral type. A typical theoretical BS catalogue gave about 75 classical Ba stars, compared with 37 observed; but no doubt some of the theoretical $\mathrm{Ba}$ red giants would be rather mildly enriched. If we change criterion (b) so that the secondary is instead a main sequence star of any temperature or luminosity, but keep the other criteria the same, we find 23 systems. Thus for every three classical Ba stars brighter than $V=6.0$, we expect one main sequence ' $\mathrm{Ba}$ star' analogue. This is perhaps a more encouraging ratio than one might have expected, given that the space density of main sequence stars is enormously greater than that of red giants.

The average theoretical Ba star contains a white dwarf of about $0.65 M_{\odot}$, and a red giant of about $1.5 M_{\odot}$ at zero age; while the average theoretical Ba-rich main sequence star contains a slightly more massive WD, with a late-B to earlyG component averaging about $1.8 M_{\odot}$ at zero age. It remains a problem why objects of the latter kind are not more commonly identified. Some possibilities (Tomkin et al.1989, Bond, private communication) are that (a) the gainer may have been set in relatively rapid rotation by the accretion process, so that the lines of enhanced metals are not conspicuous, or (b) selective diffusion may have altered the photospheric abundances to some standard abundances which are independent of the actual abundances deeper down. But neither of these is likely to be effective for the 10 or so objects expected with F or G dwarf spectra. HR 107 (Tomkin et al.1989) is a clear F5 candidate for the kind of star expected, but it is slightly fainter than $V=6$, and it does not (Jorissen, private communication) show radial velocity variation as required.

In conclusion, it is clear that the evolution of binaries which, in a conservative picture, would take place in late Case B or late Case C, is significantly modified by non-conservation, both of mass and of angular momentum. In particular, late Case $\mathrm{B}$ evolution is probably modified by the CRAP process, where the stellar wind from the cool component prior to RLOF is strongly enhanced by tidal interaction. This allows some such binaries to become cool Algols, rather than to enter a common envelope phase with probable coalescence. For the case that the initial masses are really closely equal (within $\sim 2 \%$, e.g. RZ Cha quoted earlier) we ca expect the outcome of RLOF to be an Algol-like binary of the type discussed by Popper (this conference) where both components are giants. In late Case $\mathrm{C}$ there is probably not a need for such enhancement, though it is likely nevertheless to take place, at least for the shorter-period objects in this category. Non-conservative late Case $\mathrm{C}$ seems to lead predominantly to the formation of Ba stars, although this should be via s-process-enhanced main sequence binaries for which there is still a marked shortage of candidates among stars brighter than 6th magnitude. 


\section{References}

Boffin, H.M.J. and Jorissen, A. (1988) Astron. Astrophys. 205, 155

Dominy, J.F. and Lambert, D.L. (1983) Astrophys. J. 270, 180

Eggleton, P.P., Fitchett, M.J. and Tout, C.A. (1990) Astrophys. J. 347, 998

Eggleton, P.P and Tout, C.A. (1989) in IAU Coll 107, 'Algols', ed. A.H. Batten, p 165

Griffin, R.F. (1985) in 'Interacting Binaries', ed. P.P. Eggleton and J.E. Pringle Reidel: Dordrecht p 1

Hall, D.S. (1990) in 'Active Close Binaries', ed. C. İbanoglu, p 95

Heintz, W.D. (1991) Astron. J. 101, 1071

Hoffleit, D. (1983) The Bright Star Catalogue, 4th edition; New Haven: Yale University Observatory

İbanoglu, C. (1990) in 'Active Close Binaries', ed. C. İbanoglu, p 515

Judge, P.G. and Stencel, R.E. (1991) Astrophys. J. 371, 357

Lü, P.K. (1991) Astron. J. 101, 2229

Luck, R.E. and Bond, H.E. (1982) Astrophys. J. 259, 792

Miller, G.E. and Scalo, J.M. (1979) Astrophys. J. Suppl. 41, 513

Mullan, D.J., Sion, E.M., Bruhweiler, F.C. and Carpenter, K.G. (1989) Astrophys. J. 339, L33

Paczyński, B. and Sienkiewicz, R. (1972) Acta Astron. 22, 73

Popper, D.M. (1980) Ann. Rev. Astr. Ap. 18,115

Popper, D.M. (1988) Astron. J. 96, 1040

Reimers, D. (1975) Mem. Soc. Roy. Sci. Liège 6e Ser. 8, 369

Tomkin, J., Lambert, D.L., Edvardsson, B., Gustafsson, B. and Nissen, P.E. (1989) Astron. Astrophys. 219, L15

Tout, C.A. and Eggleton, P.P. (1988a) Mon. Not. Roy. Astro. Soc. 231, 823

Tout, C.A. and Eggleton, P.P. (1988b) Astrophys. J. 334, 357 